\title{
Polizisten als »Straßenarbeiter« und Polizeiforschung
}

\begin{abstract}
Zugleich Besprechung von Wesley G. Skogan (Hrsg.), To Better Serve and Protect: Improving Police Practices. The Annals of The American Academy of Political and Social Sciences, Volume 593, May 2004 sowie von Edward Conlon, Blue Blood, Riverhead Books, New York 2004
\end{abstract}

W arum freut sich ein Polizist, wenn ihm nach dem Aufwachen klar wird, dass er heute gar nicht zur Dienststelle muss, sondern stattdessen zum Zahnarzt? Der Polizist, kein Masochist, leidet auch nicht an den in der Polizeiausbildung und Fachliteratur häufig genannten Ursachen für den Burn-out in Uniform. Weder empfindet er Stress durch Dienst zu ungünstigen Zeiten, noch plagen ihn Alkoholprobleme oder Teamkonflikte. Kein posttraumatisches Syndrom noch Angst vor dem polizeilichen Gegenüber lassen die anstehende Wurzelbehandlung als attraktive Alternative zum Dienst in der New Yorker Bronx erscheinen. Dort steht die Kundschaft der Polizei zwar häufig unter Drogen und sie trägt, wesentlich häufiger als bei uns, eine Schusswaffe bei sich, aber der NYPD Polizist Ed Conlon arbeitet gerne auf der Straße. Er hat Erfahrung und ist akzeptierter Mitarbeiter in einem multiethnischen Team erfolgreicher Strafverfolger. Er kennt die sozialen Brennpunkte, Szenen und Leute. Zudem hat er Humor und besitzt, für amerikanische Straßenpolizisten ungewöhnlich, einen akademischen Abschluss der Harvard University. Seine Handschrift wird aber, was seine Alma Mater betrifft, auf Personalfragebogen stets undeutlich. Man liest deshalb eher »Howard « statt Harvard. Die Berufskultur der Polizei in den USA hegt Intellektuellen gegenüber ebensoviel Misstrauen wie die bei uns und in vielen anderen Ländern. Der Polizist Conlon fühlt sich wegen seines Harvard Studiums nicht schlauer als seine Kollegen. Er beginnt jedoch über seine Erfahrungen als Polizist in New York zu schreiben -eine Art Selbsttherapie. Seine Essays sind von hoher Qualität, so dass die angesehene Zeitschrift »New Yorker « sie abdruckt, für die auch Hannah Arendt einst ihre Berichte über den Eichmann-Prozess geschrieben hat.

\section{Innere Kündigung}

Conlon geht nach etwa fünf Jahren Polizeiarbeit lieber zum Bohren als zum Dienst, weil er hilflos einem inkompetenten Sergeant und einem despotischen Captain ausgeliefert ist. Letzterer schneidet ihn wann immer er kann, vor allem dadurch, dass wochenlang vorbereitete Strafver- folgungsmaßnahmen von Conlon und seinen Kollegen kein grünes Licht kriegen. Törichte Vorgesetzte zerstören Arbeit und behandeln ihre Mitarbeiter wie Heimzöglinge auf einem Ausflug in die Fußgängerzone: Tu dies nicht, tu das nicht, was hast Du schon wieder angestellt? Dies wird in der vordersten Front der Polizeiarbeit sicherlich ebenso zum Anlass für den mentalen Ausstieg („die innere Kündigung «) wie die oben genannten Standarderklärungen, die im Psychologieunterricht für die Berufsmüdigkeit dieser Berufsgruppe angeführt werden. Man kriegt beim Lesen von Conlons Buch »Blue Blood« den Eindruck, dass die polizeibekannte Regel »Ober sticht Unter « im NYPD absurde Formen annehmen kann. Diese Regel ist trotz einer ständigen Beschwörung des »kollegialen Führungsstils« in der Berufskultur von deutschen Polizisten auch nicht völlig suspendiert, aber eine despotische Gängelei der Mitarbeiter wie sie in Blue Blood beschrieben wird, ist bei uns schwer vorstellbar.

\section{Der NYPD »Job«, Serpico und neuere Skandale}

Conlon ist fast zehn Jahre beim »Job«, so heißt das NYPD bei den dort tätigen Cops. Zunächst geht er bei der Housing Police Streife. Diese ist für die Projects zuständig, die Häuserblocks des sozialen Wohnungsbaus. Dort haben sich zur Zeit der Rekrutentätigkeit des Autors Mitte der 90er Jahre Familienprobleme mit Drogenabhängigkeit und Gangpräsenz zu einer explosiven Mischung überlagert. Conlons Turf erstreckt sich über 32 Gebäude mit 16 bis 21 Stockwerken. Die Housing Police wird im Rahmen der Zusammenlegung von Polizeiorganisation ebenso wie die UBahnpolizei dem NYPD zugeordnet. Conlon wechselt vom Streifendienst zur Drogenfahndung. Dort ist er über die Maßen erfolgreich, allerdings in der Sisyphusarbeit der Jagd nach Dealern. Ohne Selbstmitleid beschreibt er in der Mitte des Buches die Krise seiner beruflichen Identität, und fast wird er zur Aufgabe des Jobs genötigt. Er hat Glück, wird versetzt und fängt sich wieder. Am 10. September 2001 nimmt er seine Arbeit als Detective, also Kriminalpolizist auf, ein Schritt, der in der Hierarchie der New
Yorker Polizeiorganisation deutlich einen Aufstieg bedeutet. Einen Tag später ereilt die Katastrophe die Stadt, ihre Polizei und ihre Feuerwehr. Dieses Ereignis steht aber in der Gesamtschau der Berufsbiografie eher am Rande der Erzählung.

Der Autor befasst sich auf den 559 Seiten des Buchs nicht nur mit seinem Werdegang, mit seiner polizistenreichen Familie und seinen beruflichen Problemen und denen der Praxiskollegen. En passant, aber mit großer Detail- und Insiderkenntnis geht er durch die politischen Krisen der New Yorker Polizei. Er kontrastiert die Filmversionen von »Serpico « und »French Connection « mit den historisch belegten Abläufen und Entwicklungen; er spart weder die Korruptionsskandale noch die neueren Fälle Louima und Diallo aus. Die Auseinandersetzung mit diesen Vertrauenskrisen sind parteiliche, gleichwohl oft aufschlussreiche Passagen. Sie zeigen, dass es »das « NYPD nicht gibt, sondern sehr unterschiedliche Abteilungen und Menschen. Conlon und seine Kollegen hoffen im Fall Louima, der in der Wache durch den NYPD Polizisten Volpe sexuell misshandelt wird, dass dies »doch einfach nicht wahr sein könne«. Auch im Fall Diallo, ein unbewaffneter Migrant, der wegen einer »falschen Bewegung « durch den Schusswaffengebrauch von NYPD Beamten faktisch durchsiebt wird, bezieht Conlon Stellung. Wenn auch sein Ausleuchten des Vorfalls, bei dem seine Kollegen ohne Vorsatz („,without malice«) einen Unschuldigen getötet haben, Bedenkenswertes enthält, gerät hier seine Kritik gegen die Medien, insbesondere die polizeikritische New York Times, in den Bereich von überzogener Polemik. Der Argumentation Conlons beim Thema "racial profiling «, also der subtilen oder handfesten Diskriminierung durch Polizei wegen Hautfarbe oder ethnischer Herkunft, fehlt trotz humanistischer und faktisch belegbarer Einsichten schlicht die Distanz. Der »War on Drugs « ist an der Hauptkampflinie ein erbarmungs- und sinnloser Krieg gegen Angehörige von diskriminierten Minderheiten, nicht gegen Wohlstandskiffer und prominente Koksschnupfer. Viele der Alltagskonflikte zwischen Polizei und Gegenüber rühren aus der Unnachgiebigkeit politischer und moralischer Positionen. In umge- 
kehrter Weise ist die »Heiligkeit« des Rechts eines amerikanischen Bürgers auf seine Schusswaffe ein Umstand, dessen unheilige Folgen neben Opfern von Gang- und Dealerkonflikten auch die Polizei zu gewärtigen hat. Ohne allgegenwärtige legale und illegale Schusswaffen, würde Diallo genauso leben wie unzählige Polizeibeamte, auf die vom Gegenüber geschossen wurde.

Die Konflikte um das NYPD, die Unnachgiebigkeit der New York Times Berichterstattung sowie die (ja nur gelegentliche) Unentschlossenheit von Politik und polizeilicher Führung sowie ihr stetiges Bestreben, die Verantwortung für cause celèbré Brutalität von Polizisten den Charakterschwächen einzelner Beamter zuzuschreiben, erscheinen aus der Warte des "anständigen Praktikers « Edward Conlon ihrerseits als Belege der moralischen Verderbtheit derer »da oben « im Rathaus, in den Redaktionsstuben und in der liberalen Elite der Bürgerrechtler. Die Wahrheit ist komplexer. Harvard-Bildung schützt Autoren nicht vor gelegentlich betriebsblinden Argumenten, wenn sie verärgert sind. Ansonsten liest sich der Text über lange Strecken wie ein ausführliches, durch geschickte Rückblenden nur selten anstrengendes narratives Interview. Gleichzeitig bietet er für Kriminologie und Polizeiforschung eine lebendige zeitgeschichtliche Analyse und Organisationssoziologie der New Yorker Polizei. Blue Blood ist bei der Penguin Verlagsgruppe in hoher Auflage erschienen und wurde in der New York Times ausführlich besprochen. Der Stil ist für Polizei-Praktikergeschichten typisch: lakonisch und eher entdramatisierend. In Deutschland gibt es kein vergleichbares Buch, und es besteht wenig Aussicht darauf, dass in Bälde eines erscheinen wird.

\section{Polizeiforschung diesseits und jenseits des Atlantiks}

Die Polizei, ihr Zustand und ihre Anpassung an veränderte Zeiten und soziale Bedingungen sowie ihre Einbindung in demokratische Prozesse sind in Deutschland weder ein Medienthema noch ein klar ausgewiesener Forschungsgegenstand von Sozialwissenschaft oder Kriminologie. Es gibt kaum eine Tradition in der deutschen polizeisoziologischen Forschung (vgl. Ohlemacher 1999). Die wissenschaftliche Auseinandersetzung mit dem Thema Polizei ist in Einzelstudien mit unterschiedlichen Fragestellungen erfolgt. Die Studien der letzten 15 Jahre haben zwar interessante empirische Kenntnisse über die Polizei hervorgebracht, allerdings konzentrieren sie sich auf spezifische Einzelaspekte polizeilichen Handelns: etwa auf kriminalpolizeiliche Erkenntnismethoden (Reichertz 1991, Reichertz/Schröer 1992), auf Vernehmungen (Schröer 1992), auf Fremdenfeindlichkeit (Bornewasser 1996, Backes u.a. 1997) oder auf Geschlecht (Behr 2000; Müller/Müller-Franke et al. 2004). Aktuell sind Bestrebungen auszumachen, einzelne Forschungsrichtungen zusammen zu führen: Liebl/Ohlemacher (2000) verbinden polizeiinterne und polizeiexterne Forschungsansätze, die sowohl auf wissenschaftliche wie auf praxisrelevante Interessen zielen, zu einem Forschungskonzept (oder eher einer Idee davon) für und über die Polizei. Reichertz/Schröer (2003) kombinieren die hermeneutische Wissenssoziologie mit ihrem empirischen Interesse an der Polizei zu einer hermeneutischen Polizeiforschung. Und schließlich versucht Lange (2003) Polizeiforschung und Polizeiwissenschaft als Forschungszweig zum Thema Innere Sicherheit zu etablieren. Eine Auseinandersetzung mit dem Thema »Poli-

zei, Politik und Demokratie« findet dabei nicht statt. Allein der im vorigen Jahr erschienene Sammelband der Hamburger Studien zur Kriminologie und Kriminalpolitik (Herrnkind/Scheerer 2003), der aus unterschiedlichen Blickwinkeln die Polizei als Organisation mit Gewaltlizenz diskutiert, fordert in einzelnen Beiträgen Maßnahmen demokratischer Kontrolle. Die Erkenntnisse aus den äußerst raren State of the Art Ethnografien wie der von Astrid Jacobsen (2001) wären Fundamente für weitergehende Forschung, aber es fehlen systematische Bemühungen der zuständigen Disziplinen und Forschungsförderer. Eine systematische Debatte ist auch in den Studien der 70er und 80er Jahre nicht zu finden, allerdings werden hier vereinzelt Demokratiebezüge hergestellt.

Deutlich anders ist das in den USA, wo es neben den schreibenden Praktikern an Universitäten und Instituten gemäß der Schätzung des $\mathrm{Na}$ tional Research Council (NRC) etwa drei bis vier Hundert Forscher gibt, die sich mit polizeiwissenschaftlicher Forschung befassen. Die Forschungsförderung umfasst acht Millionen US Dollar pro Jahr. Im deutschen Fall muss man von einem Bruchteil dieses personellen und finanziellen Aufwands für die gesamte Forschung über Polizei ausgehen. In einer neuen NRC Veröffentlichung (Fairness and Effectiveness in Policing: The Evidence 2003) werden die Ergebnisse von vier Jahrzehnten amerikanischer Forschung zum Thema Polizei dargestellt. Im Zentrum steht das Handeln der Vertreter des Gewaltmonopols und vor allem, inwieweit das Verhalten der Polizei in den Städten, Gemeinden und ländlichen Regionen der Vereinigten Staaten als »fair« und »kontrolliert « bezeichnet werden kann. Dies ist auf der Ebene alltäglicher Kontakte zwischen Polizisten und Bürgern die Frage nach dem Verhältnis von Polizei zum Recht und zur Demokratie; ferner, was an der Praxis der Vertreter des Gewaltmonopols zu verbessern sei. So formuliert auch der Titel der hier besprochenen Fachzeitschrift, in der verschiedene am NRC Report beteiligte Autoren die Forschungsbilanz amerikanischer Polizeiforschung zusammenfassend beleuchten, einen Verbesserungsvorschlag. Er bezieht sich auf eben die Formel To Serve and Protect, die man in den USA (und in unseren von dort importierten Krimi- und Polizeiserien) sowohl auf den Türen von Streifenwagen als auch auf den Identification Badges von Polizisten lesen kann. So heißt der Titel der Publikation: To Better Serve and Protect: Improving Police Practices, übertragen auf die bei uns gängige Sympathieformel: »Die Polizei: Dein bald noch besserer Freund und Helfer! Wie man gute Praxis hinkriegt«. 
Die Erkenntnis, dass gutes management sowie leadership eine entscheidende Größe für das polizeiliche Alltagshandeln darstellen, zieht sich als Leitthema durch den gesamten Band. Führung muss gutes Management sein und wird verstanden als fachliche und persönliche Leitungs- und Leistungsverantwortung. In diesem Sinne »anständiges « Management formt die Basis einer demokratischen "Zurechnungsfähigkeit" (accountability) von Polizeien, diese setzt wiederum ein korrektes polizeiliches Vorgehen und einen anständigen Umgang mit Gegenüber und Opfern voraus. Obwohl dies in Demokratien common sense sein sollte wie Skogan im Vorwort schreibt, stellt seit 1970 die Forschung in den USA auf Seiten der Polizei immer wieder Verletzungen dieser Regeln der Fairness fest (zuletzt wieder 2001), vor allem gegenüber Afroamerikanern (Skogan 2004, 10).

Eine weitere Untersuchungsfrage des NRC Berichts betrifft die Kontrolle polizeilichen Fehlverhaltens und die Bekämpfung des Missbrauchs von Machtbefugnissen. Ist solche Kontrolle wirksam nur durch externe Organisationen von Bürgerrechtlern (watchdog agencies), Ombudsmänner oder "Bürgerbeschwerdestellen" (Police Complaints Authorities) zu bewerkstelligen wie dies bei uns seit Ende der 80er Jahre von polizeikritischen Autoren mit Hinweis auf eine angebliche »australische Erfahrung « behauptet wird (Brusten 2003)? Die amerikanischen Forschungsergebnisse weisen in eine andere Richtung. Der beste Weg zu einer Reform der Polizei sei ein interner (»...the surest path to police reform is an internal, managerial one «). Er schließe ein die ständige Weiterentwicklung einer herausfordernden Ausbildung der Praktiker, ihre Beaufsichtigung, interne Kontrollen der Praxis, Leistungsbewertung und konzeptionelle Anstrengungen (Skogan 2004, 9).

Der Ausgangspunkt fundierter Polizeiforschung in den englischsprachigen Demokratien, und das heißt vor allem sozialwissenschaftliche Forschung und nicht rechtsdogmatisch oder verwaltungswissenschaftlich orientierte, ist das Spannungsfeld zwischen

a) den normativen Vorgaben, also den Regeln und Gesetzen, an die sich Bürger halten müssen und ebensolchen Regeln und Gesetzen, die den Handlungsspielraum von Polizisten vorgeben, und

b) den praktischen Entscheidungssituationen, vor denen Beamte stehen.

Die Spannbreite, mit der die Beamten auf Situationen und Personen im Alltagsgeschäft reagieren, ob sie Bürger anhalten und kontrollieren, ermahnen, Maßnahmen ergreifen oder ergreifen müssen, oder auch wegschauen, wie sie wahrnehmen, entscheiden, handeln, nennt man in der englischsprachigen Polizeipraxis und der zugehörigen Forschung discretion (Opportunitätsprinzip). Moderne Polizeiforschung beginnt in den USA in den 50er Jahren durch Studien der American Bar Foundation über die Funktionsweise der amerikanischen Kriminaljustiz. »Discretion « wurde entdeckt und

Forschung über das System gab für immer ihren beschränkten Blick (im Original: blind emphasis) auf formale Gesichtspunkte und rechtswissenschaftliche Exegese auf.«(Skogan 2004, 6)

Ein dezidiertes Forschungsinteresse an discretion ist in der deutschen Diskussion kaum wahrnehmbar (vgl. auch Jasch 2004). Die Hegemonie der juristischen Optik in der Kriminologie, speziell des Strafrechts, hat aus guten verfassungsrechtlichen Gründen die dogmatische Auffassung des Legalitätsprinzips nahezu hermetisch konserviert. "Discretion « wird dementsprechend immer noch als Handeln in rechtsfreien Räumen verstanden, nicht als zwingende Notwendigkeit, im Wirrwarr der Alltagsrealität, wie sie sich Polizisten präsentiert, Prioritäten zu setzen. Angesichts ubiquitärer Ordnungsverstöße und Rechtsbrüche bei uns wie in allen westlichen Kulturen ist dies eine Position, die es zu überdenken gilt. Vielleicht ist der diesbezügliche Dogmatismus auch einer der Gründe dafür, weshalb Fremdenfeindlichkeit bei deutschen Polizisten so wenig professionell analysiert wird und ein so sperriges, tabuisiertes und vor allem durch wenig sachliche Argumente informiertes Thema bleibt. Wenn es keine discretion geben darf, kann es logischerweise auch kein racial profiling geben, treu gemäß der urdeutschen »Was nicht sein kann..." Logik. Ein Interesse an discretion und racial profiling muss beide Seiten dergleichen Medaille von polizeilicher Praxis in modernen Gesellschaften in Augenschein nehmen können, und das kann nur eine unabhängige professionelle Forschung.

\section{Ethnische Herkunft und Geschlecht}

Auf je 1000 Einwohner kommen in den USA fast zwei Polizeibeamte (sworn officers). Eine ähnlich geringe Polizeidichte hat die Schweiz, in Deutschland sind es mehr als drei, in Frankreich fast vier Polizisten auf 1000 Bürger. Im Amerika des Jahres 1975 waren dies noch 1.77 pro 1000 Bevölkerung. Dies entspricht dem internationalen Trend der Ausweitung formaler Kontrolle begleitet von einer noch massiveren Ausweitung der Zugriffsmöglichkeiten privater Sicherheitsdienste. Gleichzeitig verzeichnen moderne Kulturen eine Auflösung homogener und traditioneller Gesellschaftsstrukturen. Racial und gender diversity, also eine Repräsentanz nach ethnischen und geschlechtsspezifischen Maßstäben sollte im Personalaufbau der Polizeien ein Reflex dieser Entwicklung sein. Dies ist ein weiterer Schwerpunkt des englischsprachigen Forschungsinteresses, insbesondere die Effekte, die ein Wandel in Richtung größerer Gleichstellung bewirkt haben könnte. In den USA ist die Rate schwarzer Polizeibeamter zwischen 1973 und 1993 von 4\% auf
$11,3 \%$ gestiegen, so dass mittlerweile von einer proportionalen Repräsentanz von afroamerikanischen Polizisten gesprochen werden kann. Dies hat jedoch noch nicht notwendig eine kausale Auswirkung auf das Verhältnis zwischen der weißen Mehrheitsgesellschaft, ihrer Polizei und den diskriminierten Minderheiten. Es ist zunächst nicht mehr als ein Schritt in Richtung interner Chancengleichheit. Bei den deutschen Polizeien lässt sich die Repräsentanz von Beamten aus Migrantenfamilien und anderen Minderheiten nur in vergleichsweise minimalen Größenordnungen finden. Im Gegensatz dazu findet sich aber reichlich Alltagstheorie darüber, wie mehr migrantenstämmige Mitarbeiter in den eigenen Reihen der Polizei das Leben leichter beziehungsweise schwerer machen könnten. Es gibt für die deutsche Situation bezeichnenderweise weder brauchbare Zahlen noch Untersuchungen.

Was gender diversity, also Geschlechtergleichberechtigung angeht, so ist in den USA Polizeien in größeren Abteilungen (mit mehr als 100 Personalangehörigen) in den letzten zwanzig Jahren ein Anstieg von zwei auf knapp dreizehn Prozent $\mathrm{zu}$ beobachten. Dies betrifft female sworn officers, also in etwa dem Äquivalent unserer Vollzugsbeamtinnen, deren Rate gemäß zuverlässigerer FBIDaten von weniger als vier Prozent im Jahr 1975 auf fast zehn Prozent im Jahre 2001 gestiegen sein soll; anders betrachtet weisen im Jahr 1975 knapp mehr als ein Viertel der erfassten Polizeibehörden überhaupt keine weiblichen Vollzugsbeamten auf, im Jahre 2001 sind dies weniger als ein Prozent der Behörden. Die Situation von Frauen ist in den deutschen Polizeien in Bezug auf Chancengleichheit besser und auch gründlicher untersucht, allerdings hat man zumindest in den großen Departments der amerikanischen Großstädte den Eindruck, dort in den höheren Positionen häufiger Kolleginnen auf Chefsesseln zu sehen als bei uns. Man wird die Ergebnisse des in Kürze erscheinenden Abschlussberichts des DFGForschungsprojekts über die Situation von Frauen in der deutschen Polizei (vorläufige Ergebnisse in: Müller/Müller-Franke et al. 2004) abwarten müssen, bevor man über die Auswirkungen einer größeren Chancengleichheit auf die polizeiliche Alltagsarbeit Aussagen machen kann.

Die lakonische und ernüchternde Bilanz der amerikanischen Polizeiforschung („Effect of increases unknown «) erstreckt sich dort nicht nur auf die proportionale Zunahme von Frauen und Minderheitsangehörigen, sondern auch auf Unterschiede im Ausbildungsstand von Polizeibeamten. Eine Zunahme formaler Ausbildungsvoraussetzungen mit tertiären Bildungsabschlüssen sei zu verzeichnen und zwar von 10\% (1990) auf 32\% (2000). Ein hoher Prozentsatz der Police Departments (83\%) setzen bei Rekruten high school diplomas voraus. Diese entsprechen nicht unserem Abitur, sondern liegen im Niveau eher darunter. Vierzehn Prozent der Personalangehörigen haben College-Abschlüsse oder einen two- 
year degree (B.A.), aber auch diese entsprechen im Niveau nicht der Qualität des deutschen Fachhochschulabschlusses als Voraussetzung für den gehobenen Dienst; nur ein Prozent des Personals verfügt über einen four-year degree (Vollstudium). Dies wird zumindest dann, wenn zukünftig der Abschluss der Münsteraner Hochschule der Polizei einem universitären M.A. gleichwertig sein wird, bei uns ein deutlich höherer Prozentsatz sein. Die Empfehlungen für die Ausbildung für police officers von vor 25 Jahren seien heute noch nicht erfüllt, so das Fazit des NRC. Auch hier heißt die Antwort der Autoren E. R. Maguire und W. R. King auf die Frage nach der Auswirkung des Wandels in den Ausbildungsstandards: effects of education unknown (in: Skogan 2004, 26). Ob bei uns der gewachsene Bildungsstand von Beamtinnen und Beamten Wirkungen zeitigt, ist wohl kaum zu bezweifeln. Beschreiben und gemessen sind diese Effekte aber gleichfalls nicht.

\section{Veränderungen und Effektivität: Die Beweislage}

Mit erfrischender Offenheit werden in To Better Serve and Protect Trends und Aussagen beleuchtet, die auch bei uns durch "Experten " als Alltagstheorien in den Medien verbreitet werden, - ihre vermeintliche Triftigkeit bestätigt sich anscheinend durch stetige Wiederholung. Infolge des 11. September 2001 sei alles anders geworden, auch die Polizei. So oft man dies auch bei uns lesen und hören kann (z. B.: Magazin der Süddeutschen Zeitung Nr. 32 vom 6. August 2004: »Überall Qaida $)$, hat sich der Alltag in Mannheim oder Zwickau, in dem Polizei auftreten muss, wirklich so grundlegend geändert? Gewiss hat in Bezug auf mögliche zivile Angriffsziele die Vorbereitung auf große Schadensfälle (disaster planning) in westlichen Großstädten an Bedeutung gewonnen. Sicherlich widmet man islamistischen Aktivitäten, insbesondere möglichen Terrorzellen, eine intensivere Beachtung als zuvor, aber eine Militarisierung der Gewaltmonopols wie sie die Autoren der US-Studie beschreiben (Maguire/King in: Skogan 2004, 21), lässt sich bei unseren Polizeien nicht konstatieren.

Die seit ca. zwei Jahrzehnten auch in Deutschland vorzufindende Beschwörung der »Bürgernähe« als Allheilmittel für Sicherheits- und Polizeiprobleme, community policing wird dabei in der eingedeutschten Version zur »Kommunalen Kriminalitätsprävention «, ersetzt keine Wirkungsforschung über das, was dabei herausgekommen sein könnte (vgl. auch Jasch 2003).

Weil community policing ein Schlagwort ist, das ein Potpourri unterschiedlicher Strategien beschreibt, liegt die eine große Schwierigkeit beim Versuch herauszufinden, wie es die Polizeiarbeit verändert hat, darin, exakt herauszufinden, was community policing eigentlich ist. (Maguire/King in: Skogan 2004, 23)
Die Veränderung der modernen Kultur in eine »Risiko«-Gesellschaft (nach dem 1986 erschienenen Buch des Soziologen Ulrich Beck mit dem eben diesem Titel) beziehungsweise die Veränderung sozialer Beziehungen durch »Individualisierung « war gleichfalls über längere Zeit ein medien- und kongressgängiger one size fits all Entwurf einer Zukunftstheorie, komplett mit davon abgeleiteten Annahmen wie sich dies alles auf Gewalt und soziale Kontrolle niederschlagen werde. Auch hier fehlen die Belege für die tatsächlichen Auswirkungen solch interessanter, zu breit angelegter, stellenweise wenig präziser soziologischer Mutmaßungen über künftige Mentalitäts-, Wahrnehmungs-, Kommunikations- und Handlungsveränderungen in menschlichen Gesellschaften. Die Auswirkungen des risk society policing lassen sich in den USA jedenfalls Maguire und King zufolge empirisch so gut wie nicht belegen.

Anders verhält es sich mit Veränderungen in der bürokratischen Kontrolle von Polizeitätigkeit und solchen Praktiken, die durch technische Veränderungen möglich wurden. Compstat, also der daten- und computergestützte Abgleich von Kriminalitätsentwicklung und polizeilichen Tätigkeitsnachweisen, wird seit 1994 in New York City durchgeführt. Diese Überprüfungen betreffen allerdings nur die Strafverfolgung und keine anderen Dienstleistungen, und werden wohl zum Teil von den dort »vorgeführten« Managern der örtlichen Reviere als unangenehm empfunden. Der Vergleich von Departments mit oder ohne Compstat, ergibt für die Compstat Polizeien einen klarer formulierten Handlungsauftrag (clarified mission), neu geschaffene oder verbesserte Mechanismen für interne Kontrolle (internal accountability) sowie die Einführung datengestützter Entscheidungsprozeduren. Keine Unterschiede zwischen Departments mit oder ohne Compstat gab es dieser Forschung zufolge in den Strukturen geografischer Zuordnung oder bei der organisatorischen Flexibilität dieser Polizeibehörden. Die Forschung folgert daraus, dass Compstat zwar innovativ auf das Management gewirkt, jedoch in den traditionellen Hauptaufgabenbereichen von polizeilicher Tätigkeit keine Veränderung bewirkt habe. Die Vergleichsdaten sind wohl nicht ausreichend trennscharf, denn die Autoren konstatieren, dass es bei Compstat ähnlich wie bei community policing Polizeiorganisationen gebe, die die Existenz dieser Programme in ihrem $\mathrm{Zu}$ ständigkeitsbereich abstreiten (vielleicht weil sich damit ideologische Haltungen über das »richtige « policing verknüpfen?), sie aber trotzdem in gewissen Spielformen praktizieren würden. Wesley Skogan als Herausgeber des Forschungsbändchen ist einer der wenigen US-Kri- minologen, der longitudinal die Praxis von community policing in einer Großstadt (CAPS Chicago) auswertet und man wird auf die Ergebnisse seines Abschlussberichts warten müssen, bevor man vielleicht auch über alternative Polizeiansätze das Urteil effects unknown fällen muss. Mein eigener Eindruck während zahlreicher Polizeikontakte in Chicago (1999 bis 2001) war, dass die Euphorie der Hochglanzprogramme der polizeilichen Führung, sei es über community policing oder polizeiliche Leitbilder nicht unbedingt in den Niederungen der Praxis geteilt wird. Maguire und King zitieren Kategorien, mit denen Wandel tatsächlich gemessen werden kann (im Unterschied zu schlichten Neubenennungen unter Beibehaltung des business as usual): mobility,

training, transformative, analytic, communicative,

Bei den deutschen Polizeien lässt sich die Repräsentanz von Polizeien lässt sich die Reprasentanz von in vergleichsweise minimalen Größenordnungen finden. Im Gegensatz dazu findet sich aber reichlich Alltagstheorie darüber, wie mehr migrantenstämmige Mitarbeiter in den er Polizei das Leben leichter beziehungsweise schwerer machen könnten.

die als Anglismen leicht zu verstehen, aber als Meßlatte auch bei uns ein eher ernüchterndes Bild der faktischen Weiterentwicklung der Polizei entstehen lassen würden.

Beispiele für eine wirkliche Änderung der Bedingungen in den Polizeien der amerikanischen Bundesstaaten, Städte und Bezirke sind die weitreichende Verwendung des MDT (mobile data terminal) und der DNA Analysen, ansonsten gelte, dass es wenig Beweise dafür gäbe, dass dreißig Jahre Investitionen in technische Neuerungen große Änderungen in der polizeilichen Praxis oder Effektivität produziert hätten. Als schwerwiegende Mängel im jetzigen Kenntnisstand der US-Polizeiforschung wird die Tatsache hervorgehoben, dass es keine verlässlichen Daten über Schusswaffengebrauch gäbe (Maguire/King in: Skogan 2004, 32). Genannt werden unter anderem folgende »weiße Flecken «, bei denen die verfügbaren Datensysteme wichtige Dimensionen polizeilichen Verhaltens ausblenden würden: informelle Mittel der Durchsetzung polizeilicher Ziele sowie die Hilfe, die man Bürgern alltäglich zuteil werden lasse. Ferner die Mobilisierung der Gemeinwesen, die Arbeit mit deren Bewohnern sowie das Lösen von community Problemen. Auch das Zur-Verfügung-Stellen von notwendigen Informationen als Service für andere Institutionen und andere »importierte « Dienstleistungen würden nicht oder nicht ausreichend systematisch erfasst.

D. Weisburd und J. E. Eck stellen in ihrem Beitrag die Ergebnisse von Wirkungsanalysen der 
polizeilichen Praxis dar. In ihrer Mehrheit widerlegen diese Analysen schlicht die Alltagstheorie, dass Veränderungen in der Polizeistärke über längere Zeiträume hin die Kriminalitätsraten beeinflussen und dass Standardpraktiken der polizeilichen Prävention die Kriminalitätsraten verändern würden. Auch das rasche Reagieren auf Notrufe hat keine langfristig nachweisbaren Effekte. Der einzig wichtige Faktor, der zur effektiven Verbrechensaufklärung beitragen würde, ist von der Polizei kaum zu steuern: dies sei die Anwesenheit von Zeugen oder das Vorhandensein physischer Beweismittel. Für die Gültigkeit der Annahmen über den Zusammenhang von Ursache und Wirkung im Rahmen der auch bei uns diskutierten broken windows -Theorie gäbe es kaum Belege in der Forschung und die Wirksamkeit der von sozialen Bewegungen geforderten obligatorischen Festnahme (mandatory arrest) von Misshandlungstatverdächtigen im Kontext häuslicher Gewalt ergibt in unterschiedlichen Städten widersprüchliche Ergebnisse. Dies spricht dafür, dass zusätzliche Faktoren in Augenschein genommen werden müssen, bevor man sich über kausale Wirkungszusammenhänge und Effektivität solcher Maßnahmen verbreitet.

Erfolgversprechender scheint Weisburd und Eck zufolge ein geographischer fokussierter Ansatz gegenüber Kriminalitätsproblemen zu sein. Dieser könne die polizeiliche Effektivität steigern und zu einer Reduzierung von Verbrechens- und Ordnungsproblemen beitragen (Weisburd/Eck in: Skogan 2004, 53). Crackdowns, also gezielte Strafverfolgung und Kontrolle in Szenen würden kurzfristig abschreckend wirken, während es deutliche Belege für die Effektivität von Ansätzen problemorientierter und -lösender Polizeiarbeit in sozialen Brennpunkten mit hohen Kriminalitätsraten gäbe (ebenda, 54/55, 57). Wenn Polizei in Form von Partnerschaften mit örtlichen Vereinen, Initiativen agiert und ihr eine breitere Legitimität zugesprochen wird, so würde dies deutliche Auswirkungen auf ihre Bemühungen haben, ein rechts- und sozialkonformes Verhalten der Bürger zu fördern. Ein Teil deutscher Polizeipraxis in den Großstädten geht seit längerem genau in diese Richtung, nur sind die Messungsmethoden für die Evaluation solcher Anstrengungen zumeist untauglich. Der starre Blick auf allgemeine Daten der polizeilichen Kriminalstatistik ohne die Berücksichtigung spezifischer Bedingungen der sozialen Umwelt, in der Polizeiarbeit stattfindet, kann solche Trends nicht wahrnehmen. Nur eine unabhängige Forschung, deren Optik mehr in Augenschein nehmen kann als der polizeiinterne Tunnelblick, wird entsprechende Wirkungsanalysen durchführen können. Der Erfolgsdruck, der auf polizeiinternen Erhebungen lastet, verführt häufig zu vorschnellen Kausalitätsvermutungen.

In den nachfolgenden Beiträgen von To Better Serve and Protect werden die Ergebnisse von Skogan/Meares über Regelverletzungen durch Poli- zeibeamte vorgestellt mit besonderem Augenmerk auf polizeiliche Übergriffe zum Zwecke einer Selbstjustiz. Die Todesraten durch polizeilichen Schusswaffeneinsatz werden im Hinblick auf die ethnische Herkunft/Hautfarbe der Opfer diskutiert. Daten sind schwer zugänglich, aber Departments, die der Forschung Zugang gewähren, haben in der Regel vorteilhaftere racial disparity. Es geht um unterschiedliche climates of integrity. Solche Unterschiede in der Integrität bestimmter Polizeiorganisationen ließen sich gewiss auch in Deutschland untersuchen, falls entsprechende Forschungszugänge geschaffen würden. Strafverfolgung von Drogendelikten scheint in den US Polizeien eine hohe Affinität zu Integritätsproblemen aufzuweisen, da große Mengen Geld und Drogen »im Spiel« sind. Dafür finden sich auch in Conlons Blue Blood reichlich Belege. Als wichtige Ursachen für regelwidriges Verhalten und Kriminalität, begangen durch Polizeibeamte, werden zu hohe Toleranzschwellen und eine gewisse »Trägheit des Gewissens « in der unmittelbaren Kollegenschaft sowie bei den Führungsverantwortlichen benannt. Das Problem der Kontrolle solchen Verhaltens durch die Staatsanwaltschaft ist in den USA ähnlichen Ursprungs wie bei uns: Man ist auf Polizisten als Hilfsbeamte angewiesen und soll sie gleichzeitig kontrollieren.

Schließlich werden in Tylers Beitrag Ergebnisse präsentiert, die die Legitimität (in den Augen der Bürger) polizeilichen Handelns stärken. Zusammengefasst: Je legitimer polizeiliche Maßnahmen in den Augen der davon betroffenen Bürger wahrgenommen werden (selbst wenn sie zu deren Nachteil gereichen), umso höher ist die Akzeptanz der Ordnungskraft, umso eher wird Aufforderungen auch tatsächlich Folge geleistet, und - langfristig betrachtet - umso ausgeprägter ist die Gesetzestreue dieser Menschen. Im Licht der populärer werdenden »Get Tough « Philosophien in Teilen der Kriminalpolitik erscheinen solche Forschungsergebnisse als wichtiger Diskussionsstoff für die deutsche Debatte. Besser wäre noch, man hätte wenigstens ein paar ähnlich sorgfältig erhobene Forschungsdaten auch in unserem Land zur Verfügung.

\section{Zusätzliche Literatur:}

Backes, O. u.a. (Hg.) 1997: Risikokonstellationen im Polizeialltag. Ergebnisse einer mehrperspektivischen Untersuchung zum Verhältnis von Polizei und Fremden in Konfliktsituationen. Universität Bielefeld: Institut für interdisziplinäre Konflitk- und Gewaltforschung

Behr, R. 2000: Cop Culture - der Alltag des Gewaltmonopols. Männlichkeit, Handlungsmuster und Kultur in der Polizei. Stuttgart

Bornewasser, M. 1996: Feindselig oder überfordert? Soziale und strukturelle Belastungen von Polizeibeamtinnen und -beamten im Umgang mit Fremden. in: Polizei-Führungsakademie (Hg.): Fremdenfeindlichkeit in der Polizei? Ergebnisse einer wissenschaftlichen Studie. Schriftenreihe der Polizei-Führungsakademie. Lübeck
Brusten, M. 2003: Vom Ausland lernen: Mehr 'demokratische Kontrollen der Polizei' - auch in Deutschland? in: Herrnkind, M./Scheerer, S. (Hg.): Die Polizei als Organisation mit Gewaltlinzenz. Möglichkeiten und Grenzen der Kontrolle. Hamburg: 261-282

Council of Europe (Hg.), 2000: Police Powers and Accountability in a Democratic Society. Strasbourg

Herrnkind, M./Scheerer, S. (Hg.): Die Polizei als Organisation mit Gewaltlizenz. Möglichkeiten und Grenzen der Kontrolle. Hamburg

Herrnkind, M. 2003: Möglichkeiten und Grenzen polizeilicher Binnenkontrolle. Eine Perspektive der Bürgerrechtsbewegung. in: Herrnkind, M./Scheerer, S. (Hg.): Die Polizei als Organisation mit Gewaltlizenz. Möglichkeiten und Grenzen der Kontrolle. Hamburg: 131-156

Jacobsen, A. 2001: Die gesellschaftliche Wirklichkeit der Polizei. Dissertation, Bielefeld

Jasch, M. 2003: »Kommunale Kriminalitätsprävention in der Krise « in: MschrKrim 86 (6), 411-420

Jasch, M. 2004: »Anregungen für das deutsche Strafverfahrensrecht aus England « in: NJW Heft 15, 1077-1080

Kersten, J. 2000: General Report and Recommendations. in: Council of Europe (Hg.), 2000: Police Powers and Accountability in a Democratic Society. Strasbourg, 137-144

Kersten, J. 2003: Zero Tolerance oder Community Policing. Ein Essay zur Geschichte und zum Stellenwert eines Ideologiestreits. in: Herrnkind, M./Scheerer, S. (Hg.): Die Polizei als Organisation mit Gewaltlizenz. Möglichkeiten und Grenzen der Kontrolle. Hamburg:105-122

Lange, H.-J. (Hg.) 2003: Die Polizei der Gesellschaft. Zur Soziologie der Inneren Sicherheit. Opladen

Lehne, W. 1992: Die Polizei - ein Dienstleistungsbetrieb oder Institution staatlicher Herrschaftsgewalt? in: Kriminologisches Journal, 4. Beiheft: 34-45

Liebl, K./Ohlemacher, T. (Hg.) 2000: Empirische Polizeiforschung. Interdisziplinäre Perspektiven in einem sich entwickelnden Forschungsfeld. Herbolzheim

Müller, U./Müller-Franke, W./ Pfeil, P./Wilz, S. 2004: Alles eine Frage der Zeit? Zur Situation von Männern und Frauen in der Polizei. Texte Nr. 37, Hochschule für Polizei, FH VillingenSchwenningen,

Ohlemacher, T. 1999: Empirische Polizeiforschung in der Bundesrepublik Deutschland. Versuch einer Bestandsaufnahme. Forschungsberichte Nr. 75, Kriminologisches Forschungsinstitut Niedersachsen e.V. Hannover

Reichertz, J. 1991: Aufklärungsarbeit. Kriminalpolizisten und Feldforscher bei der Arbeit. Stuttgart

Reichertz, J./Schröer, N. (Hg.) 1992: $\quad$ Polizei vor Ort. Studien zur empirischen Polizeiforschung. Stuttgart

Reichertz, J./Schröer, N. (Hg.) 2003: Hermeneutische Polizeiforschung. Opladen

Reemtsma, J.P. 2003: Organisationen mit Gewaltlizenz - ein zivilisatorisches Grundproblem. in: Herrnkind, M./Scheerer, S. (Hg.): Die Polizei als Organisation mit Gewaltlizenz. Möglichkeiten und Grenzen der Kontrolle. Hamburg: 7-23

Schröer, N. 1992: Der Kampf um Dominanz. Hermeneutische Fallanalyse einer Beschuldigtenvernehmung. Berlin, New York

Joachim Kersten ist Professor an der Polzeifachhochschule Villingen-Schwennigen und Mitherausgeber dieser Zeitschrift 